\title{
CONTRACTIFICATION OF A SEMIGROUP OF MAPS
}

\author{
HWEI-MEI KO ${ }^{1}$ AND KOK-KEONG TAN ${ }^{2}$
}

\begin{abstract}
Let $(X, \tau)$ be a metrizable topological space, $\mathscr{P}(\tau)$ be the family of all metrics on $X$ whose metric topologies are $\tau$. Assume that the semigroup $F$ of maps from $X$ into itself, with composition as its semigroup operation, is equicontinuous under some $d \in \mathscr{P}(\tau)$; then we have the following results:

I. There exists $d^{\prime} \in \mathscr{P}(\tau)$ such that $f$ is nonexpansive under $d^{\prime}$ for each $f \in F$.

II. If $F$ is countable, commutative, and for each $f \in F$, there is $x_{f} \in X$ such that the sequence $\left(f^{n}(x)\right)_{n-1}^{\infty}$ converges to $x_{f}, \forall x \in X$, then there exists $d^{\prime \prime} \in \mathcal{P}(\tau)$ such that $f$ is contractive under $d^{\prime \prime}$ for each $f \in F$.

III. If there is $p \in X$ such that (1) $\lim _{n \rightarrow \infty} f^{n}(x)=p, \forall x \in X$ and $\forall f \in F$, (2) there is a neighbourhood $B$ of $p$ such that $\lim _{m \rightarrow \infty} f_{n_{1}} f_{n_{2}} \cdots f_{n_{m}}(B)=\{p\}$ for any choice of $f_{m_{4}} \in F, i=1, \ldots, m$, and the limit depends on $m$ only, then for each $\lambda$ with $0<\lambda<1$, there exists $d^{\prime \prime \prime} \in \mathscr{P}(\tau)$ such that each $f$ in $F$ is a Banach contraction under $d^{\prime \prime \prime}$ with Lipschitz constant $\lambda$.
\end{abstract}

1. Introduction. Let $(X, d)$ be a metric space. A map $f: X \rightarrow X$ is nonexpansive if $d(f(x), f(y)) \leqslant d(x, y)$ for all $x, y \in X$. If $x \neq y \Rightarrow d(f(x), f(y))<$ $d(x, y)$, then we say $f$ is Edelstein contractive or simply contractive. Let $F$ be a family of maps on the metric space $(X, d)$. It is evident that $F$ is equicontinuous on $X$ if each $f$ in $F$ is nonexpansive. However if $F$ is equicontinuous on $X$ under $d$, one cannot claim that even a single map in $F$ is nonexpansive with respect to this metric $d$. However in the case when $F$ is a semigroup (where the semigroup operation is understood as the composition of maps), we prove that there is a metric $d^{\prime}$ equivalent to $d$ such that each map in $F$ is nonexpansive under $d^{\prime}$. Therefore the notions of equicontinuity and nonexpansiveness of a semigroup of maps are metrically equivalent in the sense that both notions are compatible under some metric which preserves the original metric topology. Furthermore, if $F$ is countable and commutative such that for each $f \in F$ the iterate sequence $\left\{f^{n}(x)\right\}$ converges to the same point for every $x \in X$, then there is a metric $d^{\prime \prime}$ equivalent to $d$ such that each $f$ in $F$ is Edelstein contractive with respect to $d^{\prime \prime}$. Finally for a not necessarily count-

Received by the editors June 27, 1978 and, in revised form, September 19, 1978.

AMS (MOS) subject classifications (1970). Primary 54E35, 54E40; Secondary 54C05, 54H25.

Key words and phrases. Contractification, nonexpansive, contractive, equicontinuous, evenly continuous, one point compactification.

${ }^{1}$ This work was done while the author was on leave at Dalhousie University from Tsing Hua University, supported by National Science Council of the Republic of China.

${ }^{2}$ This author was supported by the National Research Council of Canada under grant number A8096. 
able nor commutative semigroup of maps $F$, under an additional condition, we prove that there is a metric $d^{\prime \prime \prime}$ equivalent to $d$ such that each $f$ in $F$ is a Banach contraction with respect to $d^{\prime \prime \prime}$ with the same Lipschitz constant.

2. The contractification of a countable commutative semigroup of maps. For a metrizable topological space $(X, \tau)$, let $\mathscr{P}(\tau)$ be the family of all metrics on $X$ whose metric topologies are $\tau$.

Theorem 1. Let $(X, \tau)$ be a metrizable topological space and let $F$ be a semigroup of maps from $X$ into itself. If for some $d \in \mathscr{P}(\tau), F$ is equicontinuous on $X$ under $d$, then there exists $d^{\prime} \in \mathcal{P}(\tau)$ such that $f$ is nonexpansive under $d^{\prime}$ for each $f$ in $F$.

Proof. Without loss of generality, we may assume that the identity map $I$ is in $F$. Since $F$ is also equicontinuous under the metric $1 \wedge d,((1 \wedge d)(x, y)$ $=\min \{1, d(x, y)\})$ and $1 \wedge d \in \mathscr{P}(\tau)$, we may assume that $d$ is a bounded metric. Define

$$
d^{\prime}(x, y)=\sup \{d(f(x), f(y)): f \in F\} \quad \text { for } x, y \in X .
$$

It is easy to see that $d^{\prime}$ is a metric on $X$. If $g \in F$, we see that

$$
\begin{aligned}
d^{\prime}(g(x), g(y)) & =\sup \{d(f(g(x)), f(g(y))): f \in F\} \\
& \leqslant \sup \{d(h(x), h(y)): h \in F\}=d^{\prime}(x, y),
\end{aligned}
$$

so that $\boldsymbol{g}$ is nonexpansive with respect to $d^{\prime}$ for each $g \in F$. It remains to show that $d^{\prime}$ is equivalent to $d$. As $d^{\prime}(x, y)>d(x, y)$ for any $x, y \in X$, it suffices to show that for any sequence $\left(x_{n}\right)_{n=1}^{\infty}$ in $X$ and $x \in X, d\left(x_{n}, x\right) \rightarrow 0$ as $n \rightarrow \infty$ implies $d^{\prime}\left(x_{n}, x\right) \rightarrow 0$ as $n \rightarrow \infty$. Indeed, let $\varepsilon>0$ be arbitrarily given. Since $F$ is equicontinuous at $x$, there exists $\delta>0$ such that

$$
d(y, x)<\delta \Rightarrow d(f(y), f(x))<\varepsilon / 2, \quad \forall f \in F .
$$

Since $d\left(x_{n}, x\right) \rightarrow 0$ as $n \rightarrow \infty$, there is a positive integer $N$ such that $d\left(x_{n}, x\right)$ $\left\langle\delta, \forall n>N\right.$. But then for each $n \geqslant N, \exists f_{n} \in F$ such that

$$
d^{\prime}\left(x_{n}, x\right)<d\left(f_{n}\left(x_{n}\right), f_{n}(x)\right)+\varepsilon / 2 .
$$

It follows from (*) and (**) that $\forall n \geqslant N$,

$$
d^{\prime}\left(x_{n}, x\right)<d\left(f_{n}\left(x_{n}\right), f_{n}(x)\right)+\varepsilon / 2<\varepsilon / 2+\varepsilon / 2=\varepsilon .
$$

Hence $d^{\prime}\left(x_{n}, x\right) \rightarrow 0$ as $n \rightarrow \infty$

In particular, if we take $F$ in Theorem 1 to be the iterates $\left\{f^{n}: n=1\right.$, $2, \ldots\}$ of a single map $f$, then we have:

COROllaRY 1.1. Let $(X, \tau)$ be a metrizable topological space and let $f$ be a map on $X$ to itself. If for some $d \in \mathcal{P}(\tau)$ the iterate sequence of maps $\left(f^{n}\right)_{n=1}^{\infty}$ is equicontinuous under $d$, then there exists $d^{\prime} \in \mathcal{P}(\tau)$ such that $f$ is nonexpansive under $d^{\prime}$. 
Theorem 2. Let $(X, \tau)$ be a metrizable topological space and let $F$ be a semigroup of maps from $X$ into itself which is equicontinuous under some $d \in \mathscr{P}(\tau)$. Let $f \in F$ be such that

(1) $f$ commutes with each $g$ in $F$,

(2) for some $a \in X$, the sequence $\left(f^{n}(x)\right)_{n=1}^{\infty}$ converges to a for each $x \in X$.

Then there exists a metric $d^{\prime}$ in $\mathscr{P}(\tau)$ such that $f$ is contractive and each $g$ in $F$ is nonexpansive under $d^{\prime}$.

Proof. By Theorem 1 there is a metric $d \in \mathscr{P}(\tau)$ such that each $g$ in $F$ is nonexpansive under $d$. Now define

$$
d^{\prime}(x, y)=\sum_{n=0}^{\infty} \frac{1}{2^{n}} d\left(f^{n}(x), f^{n}(y)\right) \quad \text { for } x, y \in X,
$$

where $f^{0}$ is the identity map on $X$. Clearly $d^{\prime}$ is a metric on $X$ and $d^{\prime}$ is equivalent to $d$ because $d \leqslant d^{\prime} \leqslant 2 d$. Also each $g \in F$ is $d^{\prime}$-nonexpansive, since

$$
\begin{aligned}
d^{\prime}(g(x), g(y)) & =\sum_{n=0}^{\infty} \frac{1}{2^{n}} d\left(f^{n}(g(x)), f^{n}(g(y))\right) \\
& =\sum_{n=0}^{\infty} \frac{1}{2^{n}} d\left(g\left(f^{n}(x)\right), g\left(f^{n}(y)\right)\right) \\
& <\sum_{n=0}^{\infty} \frac{1}{2^{n}} d\left(f^{n}(x), f^{n}(y)\right)=d^{\prime}(x, y), \quad \forall x, y \in X .
\end{aligned}
$$

To prove that $f$ is contractive with respect to $d^{\prime}$, let $x, y \in X$ with $x \neq y$. Suppose $d^{\prime}(f(x), f(y))=d^{\prime}(x, y)$. Then by the definition of $d^{\prime}$, we have

$$
d\left(f^{n+1}(x), f^{n+1}(y)\right)=d\left(f^{n}(x), f^{n}(y)\right), \quad \forall n=0,1,2, \ldots
$$

This implies $d\left(f^{n}(x), f^{n}(y)\right)=d(x, y) \neq 0, \quad \forall n=1,2, \ldots$, which contradicts the fact that $f^{n}(x) \rightarrow a$ and $f^{n}(y) \rightarrow a$. Hence $f$ is contractive under $d^{\prime}$.

The following definition can be found in [3].

Definition. Let $F$ be a family of maps on a topological space $X$ to a topological space $Y . F$ is evenly continuous on $X$ if for each $x \in X$, each $y \in Y$ and each neighbourhood $U$ of $y$, there is a neighbourhood $V$ of $x$ and a neighbourhood $W$ of $y$ such that $f(V) \subset U$ whenever $f(x) \in W, f \in F$.

It is clear from the definition that if $F$ is equicontinuous under some $d \in \mathscr{P}(\tau)$, then $F$ is evenly continuous.

Corollary 2.1. Let $(X, \tau)$ be a metrizable topological space and $f: X \rightarrow X$. Assume

(1) there exists $a \in X$ such that the sequence $\left(f^{n}(x)\right)_{n=1}^{\infty}$ converges to a for each $x \in X$,

(2) the family $\left\{f^{n}: n=1,2, \ldots\right\}$ is evenly continuous on $X$.

Then there exists $d^{\prime} \in \mathcal{P}(\tau)$ such that $f$ is contractive under $d^{\prime}$. 
Proof. It follows from Theorem 2 and the fact that (1) and (2) imply the equicontinuity of $F=\left\{f^{n}: n=1,2, \ldots\right\}$ under any $d \in \mathscr{P}(\tau)$ (see [3]).

Corollary 2.2. Let $(X, \tau)$ be a metrizable topological space and $f: X \rightarrow X$. Assume that the sequence $\left(f^{n}(x)\right)_{n=1}^{\infty}$ converges for each $x \in X$, and the family $\left\{f^{n}: n=1,2, \ldots\right\}$ is evenly continuous. Then the following are equivalent:

(1) there exists $d^{\prime} \in \mathcal{P}(\tau)$ such that $f$ is contractive under $d^{\prime}$;

(2) there exists $a \in X$ such that the sequence $\left(f^{n}(x)\right)_{n=1}^{\infty}$ converges to a for each $x \in X$;

(3) for any nonempty compact f-invariant subset $Y$ of $X, \cap_{n=1}^{\infty} f^{n}(Y)$ is a singleton.

Proof. The implication $(3) \Rightarrow(2)$ is trivial under the assumption that $\left(f^{n}(x)\right)_{n=1}^{\infty}$ converges for each $x \in X$ and that $f$ is continuous. Corollary 2.1 shows that $(2) \Rightarrow(1)$. To prove $(1) \Rightarrow(3)$, let $Y$ be a nonempty compact $f$-invariant subset of $X$. Set $A=\bigcap_{n=1}^{\infty} f^{n}(Y)$. Then $f$ maps $A$ onto $A$. As $f(A)$ is compact, there exists $x, y \in A$ such that $d^{\prime}(f(x), f(y))$ is the diameter $\delta(f(A))$ of $f(A)$ under $d^{\prime}$. Suppose $x \neq y$, then

$$
\delta(f(A))=d^{\prime}(f(x), f(y))<d^{\prime}(x, y)<\delta(A) .
$$

This contradicts the fact that $f(A)=A$. Hence $\delta(f(A))=0$, i.e., $A$ is a singleton.

REMARKS. (i) Note that the assumptions in Corollary 2.2 are equivalent to that $\left\{f^{n}: n=1,2, \ldots\right\}$ is equicontinuous under any $d \in \mathscr{P}(\tau)$ and $\left(f^{n}(x)\right)_{n=1}^{\infty}$ converges for each $x \in X$.

(ii) The assumption that $\left\{f^{n}: n=1,2, \ldots\right\}$ is evenly continuous in Corollary 2.2 cannot be weakened to the condition that $f$ is continuous without affecting the equivalences of (1), (2) and (3). In fact, the counterexample in [2] shows that (1) and (3) are not equivalent if $\left\{f^{n}: n=1,2, \ldots\right\}$ is not equicontinuous. This means that the main result in [1] is false. The following example shows that (2) and (3) are not equivalent if $\left\{f^{n}: n=1\right.$, $2, \ldots\}$ is not equicontinuous.

EXAMPLE. Let $X$ be the set of all integers equipped with discrete topology, and let $X^{*}=X \cup\{\infty\}$ be the one point compactification of $X$. Define $f$ : $X^{*} \rightarrow X^{*}$ as follows: $f(n)=n+1, \forall n \in X$, and $f(\infty)=\infty$. Then $f$ is continuous on $X^{*}$ such that $f^{n}(x) \rightarrow \infty$ as $n \rightarrow \infty, \forall x \in X^{*}$. Note that $X^{*}$ is metrizable since it is a Hausdorff space with a countable base. It is evident that $\cap_{n=1}^{\infty} f^{n}\left(X^{*}\right)=X^{*}$. So now we have the continuous map $f$ on the compact metrizable topological space $X^{*}$ such that (2) of Corollary 2.2 is satisfied but not (3). The crucial point is that the family $\left\{f^{n}: n=1,2, \ldots\right\}$ is not equicontinuous at the point $\infty$, although it is equicontinuous at any other point of $X^{*}$.

Now we come to our main object of this section. 
TheOREM 3. Let $(X, \tau)$ be a metrizable topological space, and let $F$ be a countable commutative semigroup of maps from $X$ into itself. If $F$ satisfies the conditions

(1) for each $f \in F$, there exists $x_{f} \in X$ such that the sequence $\left(f^{n}(x)\right)_{n=1}^{\infty}$ converges to $x_{f}$, for all $x \in X$,

(2) $F$ is equicontinuous under some $d \in \mathscr{P}(\tau)$,

then there exists $d^{\prime} \in \mathcal{P}(\tau)$ such that $f$ is contractive under $d^{\prime}$ for each $f \in F$.

Proof. We may write $F=\left\{f_{1}, f_{2}, f_{3}, \ldots\right\}$ for it is countable. Apply Theorem 2 to each map $f_{n}$; we get a metric $d_{n} \in \mathcal{P}(\tau)$ such that $f_{n}$ is contractive under $d_{n}$ and $f_{k}$ is nonexpansive under $d_{n}$ for each $k>1$. Now we have a sequence of metrics $d_{n}$ in $\mathcal{P}(\tau)$, from which we define

$$
d^{\prime}(x, y)=\sum_{n=1}^{\infty} \frac{1}{2^{n}} \frac{d_{n}(x, y)}{1+d_{n}(x, y)}, \quad \forall x, y \in X .
$$

It is clear that $d^{\prime}$ is a metric on $X$. By the special property of the real valued function $g(r)=r /(1+r), r \geqslant 0(g(r)$ is strictly increasing and $g(r) \rightarrow 0 \Leftrightarrow r$ $\rightarrow 0$ ), one can prove that $d^{\prime} \in \mathscr{P}(\tau)$. Each $f_{k}$ in $F$ is nonexpansive under $d^{\prime}$, for

$$
\begin{aligned}
d^{\prime}\left(f_{k}(x), f_{k}(y)\right) & =\sum_{n=1}^{\infty} \frac{1}{2^{n}} \frac{d_{n}\left(f_{k}(x), f_{k}(y)\right)}{1+d_{n}\left(f_{k}(x), f_{k}(y)\right)} \\
& \leqslant \sum_{n=1}^{\infty} \frac{1}{2^{n}} \frac{d_{n}(x, y)}{1+d_{n}(x, y)}=d^{\prime}(x, y) .
\end{aligned}
$$

Next we prove that $f_{k}$ is contractive under $d^{\prime}$ for each $k>1$. Suppose on the contrary that some $f_{k}$ is not contractive under $d^{\prime}$. Then there exist $x, y$ in $X$, $x \neq y$, such that $d^{\prime}\left(f_{k}(x), f_{k}(y)\right)=d^{\prime}(x, y)$. This implies

$$
\frac{d_{n}\left(f_{k}(x), f_{k}(y)\right)}{1+d_{n}\left(f_{k}(x), f_{k}(y)\right)}=\frac{d_{n}(x, y)}{1+d_{n}(x, y)}, \quad \forall n=1,2, \ldots,
$$

which in turn implies $d_{n}\left(f_{k}(x), f_{k}(y)\right)=d_{n}(x, y), \forall n=1,2, \ldots$, and, in particular, that $d_{k}\left(f_{k}(x), f_{k}(y)\right)=d_{k}(x, y)$. This contradicts the fact that $f_{k}$ is contractive under $d_{k}$. Hence each $f_{k}$ in $F$ is contractive under $d^{\prime}$. This completes the proof.

\section{The uniform contractification of a semigroup of maps.}

TheOREM 4. Let $(X, \tau)$ be a metrizable topological space, let $F$ be a semigroup, not necessarily commutative, of maps from $X$ into itself, and let $p \in X$. Assume that $F$ satisfies the following conditions:

(1) $\lim _{n \rightarrow \infty} f^{n}(x)=p, \forall x \in X$ and $f \in F$;

(2) there exists a neighbourhood $B$ of $p$ such that $\lim _{m \rightarrow \infty} f_{n_{1}} \cdots f_{n_{m}}(B)=$ $\{p\}$ for any choice of maps $f_{n_{i}} \in F, i=1, \ldots, m$, and the limit depends on $m$ only; 
(3) $F$ is equicontinuous under some $d \in \mathscr{P}(\tau)$.

Then for each $\lambda$ with $0<\lambda<1$, there exists a metric $d^{\prime} \in \mathcal{P}(\tau)$ such that each $f \in F$ is a Banach contraction with Lipschitz constant $\lambda$.

Proof. Apply Theorem 1 to get a metric $d \in \mathscr{P}(\tau)$ such that each $f \in F$ is nonexpansive under $d$. By (2) we may assume $B$ to be a $d$-open ball with centre $p$ and satisfying condition (2). Then $B$ is invariant under each $f \in F$. For each positive integer $m$, define

$$
\text { (4) } \begin{aligned}
A_{0}=\{B\}, A_{m} & =\left\{f_{n_{1}} \cdots f_{n_{m}}(B): f_{n_{1}}, \ldots, f_{n_{m}} \in F\right\}, \\
A_{-m} & =\left\{\left(f_{n_{1}} \cdots f_{n_{m}}\right)^{-1}(B): f_{n_{1}}, \ldots, f_{n_{m}} \in F\right\} .
\end{aligned}
$$

Let $I$ be the set of all integers. Then we have

(5) $A_{m+1}$ is a refinement of $A_{m}$ for $m \in I$;

(6) $\cup_{m \in I} A_{m}^{0}$ is an open covering of $X$, where $A_{m}^{0}=$ the interior of $A$ : $\left.A \in A_{m}\right\}$;

(7) $\lim _{m \rightarrow \infty} d\left[\cup_{A \in A_{m}} A\right]=0$, where $d[\mathcal{C}]$ is the $d$-diameter of the set $\circlearrowright$.

It is evident that (5) and (7) follow from (4) and (2), respectively. (6) follows from (1) and the fact that $A_{m}$ is a family of open sets for $m<0$. For $x, y \in X$, let $\delta(x, y)$ be the set of all possible finite sequences $\left(\left\langle x_{i}, n_{i}\right\rangle\right)_{i=0}^{m}$ in $X \times I$ where $x_{0}=x, x_{m}=y$ and for each $i=1, \ldots, m,\left\{x_{i-1}, x_{i}\right\} \subset A$ for some $A \in A_{n_{i}}$. Let $\lambda, 0<\lambda<1$, be given. For $x, y \in X$ we define

$$
d^{\prime}(x, y)=\inf _{\delta(x, y)} \sum_{i=1}^{m} \lambda^{n_{i}} d\left(x_{i-1}, x_{i}\right)
$$

where the infimum is taken over all members $\left(\left\langle x_{i}, n_{i}\right\rangle\right)_{i=0}^{m}$ in the set $\delta(x, y)$. As $\mathcal{S}(x, y)$ is nonempty for each $x, y \in X, d^{\prime}$ is well defined on $X \times X$. It is easy to check that $d^{\prime}$ is a pseudometric on $X$. We claim that

(8) $d^{\prime}(f(x), f(y))<\lambda d^{\prime}(x, y), \forall f \in F$ and $x, y \in X$.

To prove this, let $\left(\left\langle x_{i}, n_{i}\right\rangle\right)_{i=0}^{m}$ be a member of $\delta(x, y)$. Then $\left(\left\langle f\left(x_{i}\right), n_{i}+\right.\right.$ $1\rangle)_{i=0}^{m}$ is a member of $\mathcal{S}(f(x), f(y))$. By the definition of $d^{\prime}$ we have

$$
d^{\prime}(f(x), f(y))<\sum_{i=1}^{m} \lambda^{n_{i}+1} d\left(f\left(x_{i-1}\right), f\left(x_{i}\right)\right)<\lambda \sum_{i=1}^{m} \lambda^{n_{1}} d\left(x_{i-1}, x_{i}\right),
$$

and hence

$$
d^{\prime}(f(x), f(y))<\lambda \inf _{\delta(x, y)} \sum_{i=1}^{m} \lambda^{m_{i}} d\left(x_{i-1}, x_{i}\right)=\lambda d^{\prime}(x, y) .
$$

It remains to prove that $d^{\prime}$ is a metric and is equivalent to $d$. To this end we prove

(9) $d^{\prime}<\lambda^{n} d$ on $A_{n}$ for all $n \in I$, and

(10) $d<\lambda^{-n} d^{\prime}+d\left[\cup_{A \in A_{n}} A\right]$ for each $n \in I$.

One sees that (9) follows from the definition of $d^{\prime}$ and (10) is equivalent to

(11) $\lambda^{n} d(x, y)<\sum_{i=1}^{m} \lambda^{n_{i}} d\left(x_{i-1}, x_{i}\right)+\lambda^{n} d\left[\cup_{A \in A_{n}} A\right], \forall n \in I$, for each member $\left(\left\langle x_{i}, n_{i}\right\rangle\right)_{i=0}^{m}$ in $\delta(x, y)$.

To prove (11), let $\left(\left\langle x_{i}, n_{i}\right\rangle\right)_{i=0}^{m}$ be a member of $\delta(x, y)$ and $n \in I$. We 
consider the following two cases:

(I) Suppose $x_{i} \notin A$ for all $i=0, \ldots, m$ and all $A \in A_{n}$.

Then from (5) we see that $n_{i}<n, \forall i=0, \ldots, m$, and hence

(12) $\lambda^{n}<\lambda^{n_{i}}, \forall i=0, \ldots, m$. Thus

$$
\lambda^{n} d(x, y) \leqslant \sum_{i=1}^{m} \lambda^{n} d\left(x_{i-1}, x_{i}\right)<\sum_{i=1}^{m} \lambda^{n} d\left(x_{i-1}, x_{i}\right) .
$$

Hence (11) holds in this case.

(II) Suppose $x_{i} \in A$ for some $i$ and some $A \in A_{n}$.

Let $j$ be the smallest integer and $k$ be the largest integer such that $x_{j}, x_{k}$ satisfy statement (II), and let $L=\{1,2, \ldots, j, k+1, \ldots, m\}$. Then

$$
\begin{aligned}
\lambda^{\prime \prime} d(x, y) & <\lambda^{n} \sum_{i \in L} d\left(x_{i-1}, x_{i}\right)+\lambda^{n} d\left(x_{j}, x_{k}\right) \\
< & \left.\sum_{i \in L} \lambda^{n_{i}} d\left(x_{i-1}, x_{i}\right)+\lambda^{n} d\left[\bigcup_{A \in A_{n}} A\right] \quad \text { (because (12) holds for } i \in L\right) \\
< & \sum_{i=1}^{m} \lambda^{n_{i}} d\left(x_{i-1}, x_{i}\right)+\lambda^{n} d\left[\bigcup_{A \in A_{n}} A\right] .
\end{aligned}
$$

Hence (11) also holds in this case. Therefore (10) is true for all $n \in I$. From (10) we see that if $d^{\prime}(x, y)=0$, then $d(x, y)<d\left[\cup_{A \in A_{n}} A\right]$ for all $n \in I$. Letting $n \rightarrow \infty$, we have $d(x, y)=0$ and hence $x=y$. This shows that $d^{\prime}$ is a metric on $X$. To show that $d^{\prime}$ is equivalent to $d$, let $\left(x_{k}\right)_{k=1}^{\infty}$ be a sequence in $X$ and $x_{0} \in X$. First assume $d\left(x_{k}, x_{0}\right) \rightarrow 0$ as $k \rightarrow \infty$. From (6) $\exists n \in I$ such that $x_{0} \in$ the interior of $A$ for some $A \in A_{n}$. As $x_{k} \rightarrow x_{0}, \exists N \in I$ such that $k>N \Rightarrow x_{k} \in A \in A_{n}$. From (9) we have $k \geqslant N \Rightarrow d^{\prime}\left(x_{k}, x_{0}\right)<\lambda^{n} d\left(x_{k}, x_{0}\right)$. Hence $d^{\prime}\left(x_{k}, x_{0}\right) \rightarrow 0$ as $k \rightarrow \infty$. Conversely, let $d^{\prime}\left(x_{k}, x_{0}\right) \rightarrow 0$, as $k \rightarrow \infty$. Then from (10) we have

(13) $d\left(x_{k}, x_{0}\right)<\lambda^{-n} d^{\prime}\left(x_{k}, x_{0}\right)+d\left[\cup_{A \in A_{n}} A\right], \forall n \in I$ and any positive integer $k$. Given $\varepsilon>0$, from (7) and $d^{\prime}\left(x_{k}, x_{0}\right) \rightarrow 0$ as $k \rightarrow \infty, \exists$ positive integers $N, M$ such that $d\left[\cup_{A \in A_{N}} A\right]<\varepsilon / 2$ and $d^{\prime}\left(x_{k}, x_{0}\right)<(\varepsilon / 2) \lambda^{N}, \forall k>$ $M$. Then from (13) we have

$$
d\left(x_{k}, x_{0}\right) \leqslant \frac{\varepsilon}{2}+\frac{\varepsilon}{2}=\varepsilon, \quad \forall k>M .
$$

This shows $d\left(x_{k}, x_{0}\right) \rightarrow 0$ as $k \rightarrow \infty$. Therefore $d^{\prime} \in \mathscr{P}(\tau)$ and (8) holds. This completes the proof.

COROllary 3.1. Let $(X, \tau)$ be a metrizable topological space, $F$ be a semigroup of maps from $X$ into itself, and $p \in X$. Let $F$ satisfy conditions (1) and (2) of Theorem 4 and

(14) $F$ is evenly continuous on $X$, and

(15) the closure of the set $F[x]=\{f(x): f \in F\}$ is compact for each $x \in X$.

Then for each $\lambda$ with $0<\lambda<1$, there exists $d^{\prime} \in \mathcal{P}(\tau)$ such that each $f$ in $F$ is a Banach contraction under $d^{\prime}$ with Lipschitz constant $\lambda$. 
Corollary 3.2 ([4, TheOREM (i)]). Let $(X, \tau)$ be a metrizable topological space, and let $f$ be a continuous map from $X$ into itself. Let $p \in X$ be fixed. Then for any $0<\lambda<1$, there exists $d^{\prime} \in \mathscr{P}(\tau)$ such that $f$ is a Banach contraction under $d^{\prime}$ with Lipschitz constant $\lambda$ and $f(p)=p$ if and only if $f^{n}(x) \rightarrow p$ for each $x \in X$ and

(16) $\lim _{n \rightarrow \infty} f^{n}(B)=\{p\}$ for some neighbourhood $B$ of $p$.

Proof. The necessity is obvious. The sufficiency follows from Theorem 4 by observing that if $F=\left\{f, f^{2}, \ldots\right\}$, then (16) is equivalent to (2) in Theorem 4, and furthermore, for a continuous map $f$ with $f^{n}(x) \rightarrow p, \forall x \in X$, (16) implies that $F$ is equicontinuous under some $d \in \mathcal{P}(\tau)$ (whose proof can be found in the proof of Theorem (i) in [4]).

REMARK. The main theorem in [5] states:

THEOREM. Let $(X, \tau)$ be a metrizable topological space and $f$ be a continuous self-map on $X$ such that (i) $f$ has a fixed point $p$ which has an open neighbourhood with compact closure, (ii) for every $x \in X$, the sequence $\left(f^{n}(x)\right)_{n=1}^{\infty}$ converges to $p$. Then the following statements are equivalent

(A) For each $\lambda \in(0,1)$, there exists $d_{\lambda} \in \mathcal{P}(\tau)$, complete if $X$ is topologically complete, such that $f$ is a Banach contraction under $d_{\lambda}$ with contraction constant $\lambda$.

(B) The sequence of iterates of $f$ is evenly continuous.

The implication $(\mathrm{A}) \Rightarrow(\mathrm{B})$ is trivial. We shall show that the implication (B) $\Rightarrow$ (A) can be derived from Corollary 3.2. To see this we prove that (16) holds. Let $B$ be a compact neighbourhood of $p$ (by (i)). We claim that $\lim _{n \rightarrow \infty} f^{n}(B)=\{p\}$. Let $d \in \mathscr{P}(\tau)$. Then from (ii) and (B), we see that $\left\{f, f^{2}, \ldots\right\}$ is equicontinuous under $d$. For any $\varepsilon>0$, let $U=B(p, \varepsilon)=\{y$ $\in X: d(y, p)<\varepsilon\}$. For any $x \in B, \exists \delta_{x}>0$ such that

(17) $d(y, x)<\delta_{x} \Rightarrow d\left(f^{n}(y), f^{n}(x)\right)<\varepsilon / 2, \forall n$.

From (ii), $\exists$ positive integer $N_{x}$ such that

(18) $n \geqslant N_{x} \Rightarrow d\left(f^{n}(x), p\right)<\varepsilon / 2$.

Now $\left\{B\left(x, \delta_{x}\right), x \in B\right\}$ is an open cover of $B$ which is compact, there exist $x_{i} \in B, i=1, \ldots, n$, such that $B \subset \cup_{i=1}^{n} B\left(x_{i}, \delta_{x_{i}}\right)$. Let $N=\max \left\{N_{x_{i}}: i=\right.$ $1, \ldots, n\}$. We claim that $f^{n}(B) \subset U, \forall n \geqslant N$. Indeed,

$$
\begin{aligned}
y \in B & \Rightarrow y \in B\left(x_{i}, \delta_{x_{i}}\right), \quad \text { for some } 1<i \leqslant n, \\
& \Rightarrow d\left(f^{n}(y), f^{n}\left(x_{i}\right)\right)<\frac{\varepsilon}{2}, \quad \forall n, \quad \text { by }(17), \\
& \Rightarrow d\left(f^{n}(y), p\right) \leqslant d\left(f^{n}(y), f^{n}\left(x_{i}\right)\right)+d\left(f^{n}\left(x_{i}\right), p\right) \\
& <\frac{\varepsilon}{2}+\frac{\varepsilon}{2}=\varepsilon, \quad \forall n \geqslant N, \quad \text { by }(18), \\
& \Rightarrow f^{n}(y) \in U, \quad \forall n \geqslant N .
\end{aligned}
$$

Hence (16) holds. 


\section{REFERENCES}

1. L. Janos, On the Edelstein contractive mapping theorem, Canad. Math. Bull. 18 (1975), 675-678.

2. L. Janos, H.-M. Ko and K.-K. Tan, Edelstein's contractivity and attractors, Proc. Amer. Math. Soc. (to appear).

3. J. L. Kelley, General topology, Van Nostrand Company Inc., Princeton, N. J., 1955.

4. S. Leader, A topological characterization of Banach contractions, Pacific J. Math. 69 (1977), 461-466.

5. J. L. Solomon and L. Janos, Even continuity and Banach contraction principle, Proc. Amer. Math. Soc. 69 (1978), 166-168.

Department of Mathematics, National Tsing Hua University, Hsinchu, Tarwan 300, REPUblic OF CHINA

Department of Mathematics, Dalhousie University, Halifax, Nova Scotia, Canada B3H 4H8 